\title{
Slums in den Stadtkarten des Schweizer Weltatlas
}

\section{Zum Begriff Slums}

Der Begriff Slums wurde im frühkapitalistischen Industriegebiet englischer Städte (z. B. Manchester und Liverpool) erstmals im 18. Jh. verwendet. Er bezeichnete heruntergekommene, baulich verlotterte Fabrikarbeiterwohnquartiere. Diese gehörten dem Fabrikherrn und wurden an seine Fabrikarbeiter vermietet. Wegen des doppelten Abhängigkeitsverhältnisses vom Arbeitgeber wagten die Mieter meistens nicht, Forderungen nach Sanierung zu stellen. Die meisten Bauten waren aus gebrannten Ziegelsteinen ohne Verputz erstellt. Infolge mangelnden Unterhalts und oft auch fehlender Sorgfalt durch die Mieter selbst waren sie bald baufällig und zeichneten sich auch durch einfache sanitäre Einrichtungen und Fehlen einer teureren Infrastruktur aus.

Als die Armenviertel der Städte der Dritten Welt im Laufe der 50er Jahre im Bewußtsein der Geographen Einzug hielten, wurde der Begriff Slums auch für jene meist illegal (ohne rechtmäßigen Bodenerwerb) errichteten Primitivbauten der sozial untersten Schichten am Rande von Großstädten verwendet. Diese werden durch die Bewohner selbst erstellt, meistens aus requirierten Materialien wie Holzbrettern, Wellblech, alten Autoreifen, Blechkanistern und, so man hat, Ziegel oder Dachpappe, manchmal auch geflochtenen Matten, Pavatex oder Karton. Bei der Wahl der Materialien und der Konstruktionsweise spielen auch das Klima der Region und die kulturelle Tradition eine Rolle. Im Laufe der Zeit haben sich in den verschiedenen Kulturkreisen (Sprachgebieten) folgende gebräuchliche Begriffe für slumähnliche Elendsquartiere ergeben:

\begin{tabular}{|l|l|}
\hline Sprachregion & Begriffe für slumähnliche Quartiere \\
\hline deutsch & Elendsviertel, Primitivbauten, \\
& Hüttenquartiere \\
englisch & Squatters, Slums*) \\
französisch & Bidonvilles \\
spanisch & Barriadas (Peru) \\
& Villas miserias (Argentinien) \\
portugiesisch & Callampas (Chile) = Pilze \\
Indien & Favelas \\
\hline
\end{tabular}

* Sollte eigentlich nur in seiner primären Bedeutung wie im 18. Jh. verwendet werden.
So werden in Lateinamerika die spanischen Begriffe verwendet, in Brasilien die portugiesische Bezeichnung $\mathrm{Fa}$ velas, in den ehemals französischen Kolonien der Name Bidonvilles und im englischen Einflußbereich (Indien, Hongkong) Squatters. In den Legenden der Stadtkarten des Schweizer Weltatlas wurde nicht immer der lokale Ausdruck verwendet, sondern oft dem deutschen Begriff (als Erleichterung für die unteren Schulstufen) der Vorzug gegeben.

\section{Verbreitung der Squatters und ihre kartographische Darstellung}

Squatters gehören im 20. Jahrhundert zum obligatorischen Inventar von Großstädten, insbesondere jener der Entwicklungsländer. Da sie in den Augen vieler Lokalpolitiker einen Schandfleck darstellen (man stellt Armut nicht gerne zur Schau), werden sie auf Stadtplänen nicht dargestellt oder, wenn schon kartiert, nicht als solche bezeichnet. Auch aus Statistiken über die Stadtbevölkerung können sie nicht abgeleitet werden. Somit hat der Kartograph oft zu dürftige Quellenangaben, wo genau und in welcher Ausdehnung Squatters zu finden sind. Da sich die zuletzt entstandenen Hüttenquartiere in der Regel am Stadtrand befinden und diese Regionen in der kartographischen Erfassung immer hintennachhinken, stellt die vollständige Kartierung der Squatters ein echtes Problem dar. Es gibt deshalb im Atlas auch Stadtkarten, wo die Squatters aus Gründen fehlenden Quellenmaterials nicht dargestellt wurden oder weil der Kartenautor einen andern Schwerpunkt der Karte im Auge hatte. Ferner hängt die Ausscheidung von solchen Quartieren vom Maßstab ab. Sie lassen sich im Maßstab 1:300 000 gerade noch darstellen, falls das Hüttenquartier nicht eine wesentlich kleinere Fläche als $1 \mathrm{~km}^{2}$ hat. Idealer sind Maßstäbe von 1:200000 oder größer. Es wäre also falsch, wenn der Benützer von Stadtkarten im Atlas, in welchen keine Squatters ausgeschieden wurden, glaubte, es hätte in Wirklichkeit auch keine. Als Beispiele seien die Karten von Istanbul (S. 66), Neapel (S. 67), Johannesburg-Soweto (S. 91), Kairo (S. 95), Hongkong (S. 113), New York (S. 130) und Chicago (S. 138) erwähnt; in die-

Georg Jung, Dr. phil., Kantonsschule Sargans, Rebweg 4, 7310 Bad Ragaz 


\begin{tabular}{|l|l|l|l|}
\hline Stadt & Seite & Maßstab & Bezeichnungen in der Legende \\
\hline Duala (Kamerun) & 91 & $1: 200000$ & Ältere und neuere Hüttenquartiere \\
Casablanca & 95 & $1: 100000$ & Hüttensiedlungen (Squatters) \\
Bombay & 109 & $1: 200000$ & Hüttensiedlungen (Squatters) \\
Jaipur (Indien) & 109 & $1: 200000$ & Hüttensiedlungen (Squatters) \\
Lima (Peru) & 148 & $1: 300000$ & Hüttenquartiere (Barriadas) \\
Brasilia & 148 & $1: 300000$ & Hüttenquartiere (Favelas) \\
Coyoacán (Mexiko) & 149 & $1: 50000$ & Primitivbauten \\
\hline
\end{tabular}

Tabelle 1 Liste der Stadtkarten im Schweizer Weltatlas, in denen Hüttenquartiere oder Squatters ausgeschieden sind.

sen Städten gibt es mit jeder Garantie Squatters. Falsch wäre es auch anzunehmen, europäische Städte hätten keine Slums. Diese sind vermutlich in der Fläche kleiner und eher in den wärmeren Gebieten anzutreffen. Vielleicht sind sie auch etwas solider gebaut als jene in der Dritten Welt. Manchmal sind Quartiere auch durch Verlotterung der Bausubstanz sekundär verslumt, d. h. keine echten Squatters, aber im Aussehen mit ihnen verwandt und von einer Bevölkerung bewohnt, die ebenfalls den unteren sozialen Schichten angehört. In Marseille, Paris, Neapel, Lissabon und Porto habe ich mit eigenen Augen solche Quartiere gesehen.

\begin{tabular}{|l|l|l|l|}
\hline Stadt & Jahr & $\%$ & Mio. Einw. 1990 \\
\hline Ankara & 1970 & 60 & 1,5 \\
Caracas & 1975 & 42 & 1,4 \\
Dakar & 1974 & 30 & 0,45 \\
Kalkutta & 1976 & 67 & 2,9 \\
Lima & 1974 & 36 & 2,3 \\
Mexico City & 1970 & 46 & 9,6 \\
\hline
\end{tabular}

Tabelle 2 SCHÄDLE gibt 1982 obige Werte für den Anteil derSlumbewohner an der Gesamtbevölkerung (Absolutzahl für 1990 extrapoliert).

So wie die Fläche der Hüttenquartiere nur approximativ bekannt ist, ist auch die Anzahl ihrer Bewohner und damit ihr Prozentanteil an der Gesamtbevölkerung der Stadt ebenfalls nur ungefähr abschätzbar.

Nach einer Schätzung der Weltbank wird die Stadtbevölkerung Lateinamerikas im Jahre 2000 etwa $75 \%$ der Gesamtbevölkerung betragen, also etwa 405 Mio. Nimmt man an, daß ein Drittel Slumbevölkerung ist, so wären das etwa 135 Mio. Menschen nur für Lateinamerika, die in bitterster Armut leben. Nach einer Prognose der Uno werden im Jahre $200043 \%$ oder 2,56 Mrd. Menschen der Weltbevölkerung in einer Stadt leben. Somit werden um die Jahrtausendwende schätzungsweise 860 Mio. Menschen in Squatters wohnen, wenn man einen Drittel der Stadtbevölkerung veranschlagt.

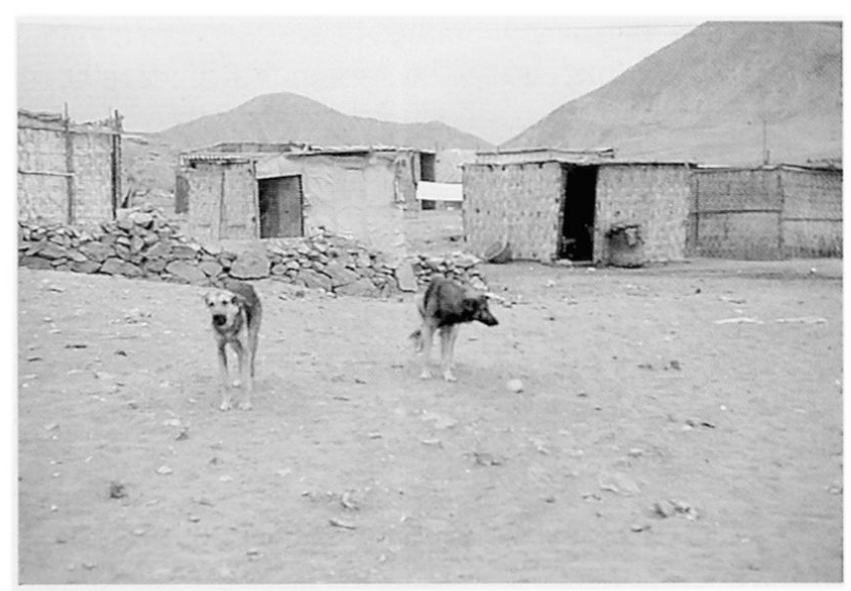

Abb. 1 Barriada "Villa el Salvador" im Süden Limas mit Primitivbauten aus geflochtenen Matten und Wellblechbedachung (siehe Karte im Atlas S. 148).

Als eine der Hauptursachen der Squatterbildung gilt die Zunahme der Landflucht und damit die Zuwanderung in die Stadt. In der Folge steigen die Arbeitslosigkeit und die Wohnungsnot, was wiederum einen Rückkoppelungseffekt hat, nämlich die Tendenz, neue Squatters zu errichten. Die Armut der vom Lande Zugewanderten erlaubt ihnen oft nicht, einen minimalen Wohnungsmietzins zu zahlen. Somit sind sie dazu verurteilt, illegale Squatters zu errichten, wo der Mietzins entfällt und der einmalige Erwerb von «Baumaterial» nicht ins Gewicht fällt, da es oft nur Abfälle sind.

\section{Anordnung und Weiterentwicklung von Squatters}

In den Karten von Lima, Brasilia und Duala kann der Schüler feststellen, daß Squatters vorwiegend am Stadtrand entstehen. Solche, die dem Zentrum näher liegen, sind früher entstanden und älter als jene an der Peripherie der Stadt. Menschen, die sich einmal in einem Slum niedergelassen haben, sind bestrebt, ihn zu verbessern. 
Möglich ist das, wenn die öffentliche Hand (Stadtbehörde) die Infrastruktur verbessert (siehe auch Hintergrundinformation bei den Aufgaben zu Lima). Als solche kommt die Verlegung von Wasserleitungen, Abwasserkanälen und Elektrizität in Betracht. Manchmal kommt der Strom auch vor der Abwasserentsorgung. Nach einer mündlichen Mitteilung eines Bewohners von Kairo hinkt die Versorgung mit Wasser und Strom oft 2-6 Jahre der Errichtung des Squatters hintennach. Später erfolgt oft eine Verbesserung der Straßen durch Asphaltierung, um die Staubbildung in der Trockenzeit und die Verschlammung in der Regenzeit zu reduzieren. Manchmal versuchen auch die Bewohner bauliche Verbesserungen vorzunehmen. Stadtbehörden bemühen sich oft, durch zwangsweise Umsiedlung Squatters zum Verschwinden zu bringen, was bei den Bewohnern der Primitivquartiere gar nicht immer auf Begeisterung stößt, wie PRONK (1995) mit einer Studie in Bangkok nachweisen konnte. Radikallösungen mit Beseitigung durch Traxfahrzeuge kommen aber immer wieder vor.

Nicht zu vergessen sind auch die Einwirkung von Feuersbrünsten oder die Schäden bei Wirbelstürmen. Die Superleichtbauweise der Squatters führt dazu, daß sie wie Zunder abbrennen oder bei Sturm wie Kartenhäuser zusammenfallen. Trotzdem besteht weltweit eine zunehmende Tendenz der Anzahl, der Gesamtfläche und der Einwohnerzahl der Squatters.

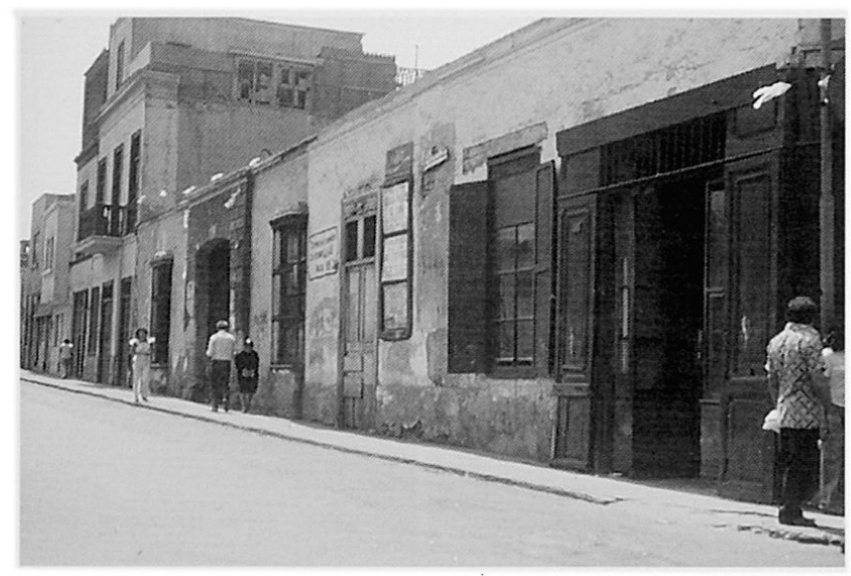

Abb. 2 Tugurios im Zentrum Limas in den 80er Jahren. Die Bauten stammen aus den 30er Jahren. Der Verputz blättert $\mathrm{ab}$, das Innere verfügt nur über einen einzigen Wasserhahn für über 50 Personen (siehe Karte im Atlas S.148).

\section{Verwandte Bildungen}

In Stadtteilen, die mehr als 100 Jahre alt sind, gibt es Quartiere, die sekundär verslumt sind, weil die einstigen Besitzer der ursprünglichen Solidbauten verarmt sind und die Bauten nicht mehr unterhalten können. Zur Gründerzeit vor 70-100 Jahren waren diese Bauten in gutem Zustand und wurden sogar von sozialem Mittelstand bewohnt. Heute fehlt das Geld, um einzelne Häuser oder oft ganze Straßenzüge oder Quartiere zu renovieren. Die Bausubstanz verlottert, und die sanitären Installationen von damals sind oft in katastrophalem Zustand. Die anspruchsvolleren und zahlungskräftigeren Mieter ziehen aus. Es rücken die sozial unteren Schichten nach, die froh sind, eine billige Wohnung gefunden zu haben. Im Atlas ist dieses Problem auf der Karte Lima dargestellt. Dort werden sekundär verslumte Stadtregionen mit einem Punktraster ausgeschieden und als "Tugurios» (= im Zerfall begriffene Quartiere) bezeichnet. Sie finden sich vorwiegend in der inneren Stadt, die vor 1940 erbaut wurde. In solchen Quartieren herrschen oft Elend und Armut, mitunter auch Prostitution und Kriminalität.

\section{Konkrete Aufgaben für den Unterricht}

\section{a) Allgemeine Probleme}

1. Der/die Schüler/in soll den Atlas nach Stadtkarten durchforsten und die Legenden daraufhin überprüfen, ob Squatters (inkl. anderssprachiger Begriffe dafür) zu finden sind. Er/sie erstellt eine tabellarische Zusammenstellung.

2. Der/die Schüler/in versucht, auf Grund der Lage der Squatters im Stadtgefüge über ihr vermutliches Alter und ihre zeitliche Reihenfolge der Gründung sich ein Bild zu machen.

\section{b) Stadt Lima, Peru (S. 148)}

1. Bestimmen der Flächenanteile der Barriadas, Tugurios und des übrigen Stadtgebietes mittels der Rastermethode (Auszählen von Rasterquadraten, die man auf einer transparenten Folie über die Karte legt). Dem Schüler macht es vielleicht weniger Mühe, die zu bestimmenden Flächen auf ein Pauspapier von der Karte durchzuzeichnen und hernach auf einen Raster zu legen. Hat man die verschiedenen Flächen ermittelt, kann man mit Hilfe der Bevölkerungskurve im Diagramm und der mittleren Wohndichte (in der Karte unten links) einigermaßen abschätzen, welcher Bruchteil der Bevölkerung und damit wie viele Bewohner in den Barriadas wohnen.

2. Welche Schlüsse läßt die Information zu, daß die Wohndichte im Zentrum von Lima $18600 \mathrm{E} / \mathrm{km}^{2}$ beträgt, in den Barriadas jedoch nur $790 \mathrm{E} / \mathrm{km}^{2}$ ?

Hintergrundinformation:

In den neuen Barriadas dauert es 1-2 Jahre, bis die Zufuhr von Wasser durch eine Leitung kommt. Vorher bringt ein Zisternenwagen täglich Trinkwasser. Nach 2-3 Jahren erfolgt die Zufuhr von elektrischer Energie, und nach 6-8 Jahren kann man mit der Asphaltierung der staubigen Straßen rechnen (Mitteilung von F. Villiger, Lima). 


\section{c) Coyoacán, Mexiko (S. 149)}

1. Wie hängen Funktion bzw. Wohnqualität, Wohndichte, Bodenpreis und Einkommenshöhe im Quartier mit Primitivbauten, die in der Karte Coyoacán dargestellt sind, miteinander zusammen?

2. Gibt es Unterschiede in der Infrastruktur oder den zentralörtlichen Diensten zwischen dem Primitivquartier und den übrigen Quartieren?

\section{d) Duala, Kamerun (S. 91)}

1. Was ist mit den ehemaligen Hüttenquartieren von 1946 beim nachträglichen Stadtwachstum geschehen?

2. In welcher Richtung hat sich die Stadt zuerst erweitert?

3. Warum wurden die neueren Hüttenquartiere (von 1983) auf ehemals landwirtschaftichem Land gebaut und nicht anderswo, z. B. südlich Bonanloka?

4. Erstelle eine Flächenberechnung der Hüttenquartiere und der ganzen Stadt (nach der unter b beschriebenen Methode).

5. Wie hat sich das Verhältnis beim Flächenanteil zwischen den Hüttenquartieren und der ganzen Stadt von 1946 zu 1983 verändert?

Hintergrundinformation:

Duala umfaßte 1983 etwa 500000 Einwohner; für 1946 wird die Einwohnerzahl auf 30000 geschätzt, da die Zählung von 1936 eine solche von 20000 ergab.

\section{e) Casablanca, Marokko (S. 95)}

1. Lokalisiere die Hüttensiedlungen.

2. Stelle eine Distanzberechnung an für den täglichen Arbeitsweg eines Hüttenquartierbewohners, der im Hafen tätig ist oder der im Hafenquartier einen Straßenhandel (z. B. Zigarettenverkauf) betreibt. Welche finanziellen Konsequenzen hat unter Umständen dieser lange Arbeitsweg?

Eine gleiche Aufgabe kann auch für Lima gelöst werden, denn dort legen viele Bewohner der Barriadas im Süden täglich den Weg in das Stadtzentrum zurück, um als illegale Händler (vendedores ilegales) mit dem Verkauf von Zigaretten, Kaugummi oder selbstverfertigtem einfachem Kunsthandwerk den bescheidenen Lebensunterhalt zu verdienen.

\section{f) Bombay - Jaipur im Vergleich (S. 109)}

1. Wie unterscheidet sich die Lage der Slums von Bombay mit jenen von Jaipur?
2. Warum droht dem Squattersgebiet in Bombay, das zwischen dem Hafen und dem westlich davon gelegenen Wohngebiet der Mittelschicht liegt, kaum Gefahr, zum Verschwinden gebracht zu werden?

3. Wo werden vermutlich neuere Squatters in Bombay entstehen?

\section{g) Brasilia, Brasilien (S. 148)}

1. Lokalisiere die Favelas in Brasília.

2. Warum fehlen in der Karte in den Satellitenstädten Guará, Sobradinho und Tagatingua die Favelas, obwohl solche nach Zeitungsberichten existent und in Entwicklung begriffen sind?

\section{Benutzte und weiterführende Literatur}

AKTUELLE IRO-LANDKARTE (1987): Karte Nr. 9/87, mit Textbeilage, Süddeutscher Verlag München.

BALDEUX, D. (1983): Entwicklungspotentiale in den Slumgebieten, Köln.

ENGELHARDT, R. (1989): Selbsthilfe und Bodenmärkte in Armensiedlungen; Fallstudie aus Salvador/Bahia, Brasilien. In: Geographische Rundschau 11/1989, Westermann, Braunschweig.

FISCHER WELTALMANACH (1995): Fischer Taschenbuchverlag, Frankfurt a. M. 1994.

GILBERT, A., WARD, P. M.(1985): Housing the state and the poor, Cambridge.

IGEL, B. (1988): Überlebensökonomie der Slumbewohner in Thailand. Diss. Freie Universität Berlin.

MORAL, H., Buenos Aires (1976): In den lateinamerikanischen Staaten werden Städte allmählich zu Slums. In: “LNN" Nr.14 vom 19.1.1976, Luzern.

NIEMEYER, G. (1969): Siedlungsgeographie. In: Das Geographische Seminar, Westermann, Braunschweig.

PRONK, M. (1995): Überlebensstrategien oder Überlebensformen? Soziale und wirtschaftliche Verflechtungen von Slumbewohnern im Klong Toey, Bangkok, Geographica Helvetica 2/1995, Zürich.

SCHÖLLER, P. (1981): Zur Urbanisierung der Erde. 43. Deutscher Geographentag.

TUNER, J.F.C. (1978): Verelendung durch Architektur; Plädoyer für eine politische Gegenarchitektur in der Dritten Welt, Reinbek.

WELTBANK (1990): Entwicklungsbericht Armut, Washington D. C.

WÜLKER, G. (1991): Der Verstädterungsprozeß in der Dritten Welt. In: OPITZ, P. (1991): Grundprobleme der Entwicklungsländer. Beck'sche Reihe Nr. 451, Beck-Verlag, München. 